\title{
Leukemic phase of hepatosplenic $T$ cell lymphoma: a case report and review of the literature
}

\author{
Elizabeth Kehr • Peter Stenzel • Gang Xu • \\ Katalin Kelemen
}

Received: 8 April 2010 /Accepted: 12 July 2010 /Published online: 21 September 2010

(C) Springer-Verlag 2010

\section{Introduction}

Hepatosplenic T cell lymphoma (HSTL) is a rare subset of peripheral $\mathrm{T}$ cell lymphoma derived from cytotoxic $\mathrm{T}$ cells usually of gamma-delta $\mathrm{T}$ cell receptor type. Clinically, HSTL is characterized by hepatosplenomegaly, absence of lymphadenopathy, cytopenias, and an aggressive clinical course with a median survival of less than 1 year. The peak incidence occurs in adolescents and young adults (median 35 years) with a male predominance. A subset of HSTL arises in the setting of chronic immune suppression, most commonly chronic immunosuppression for solid organ transplantation or immunosuppressive therapy for Crohn's disease. Histologically, HSTL preferentially infiltrates the hepatic sinusoids and splenic red pulp but also may involve lymph node sinuses, bone marrow, and peripheral blood. Hepatosplenic $\mathrm{T}$ cell lymphoma exhibits a characteristic immunophenotype that expresses both $\mathrm{T}$ cell and NK cell markers. Typical cases express CD2, CD3, CD7, CD16, CD56, gamma-delta TCR and are negative for CD4, CD8, and the alpha-beta TCR. A minority of cases appear to be of alpha-beta type, which is considered to be a variant of the more common gamma-delta form of the disease. The characteristic clinical, histologic, immunophenotypic, and cytogenetic signature of this lymphoma has led to its recognition as a distinct entity in the revised EuropeanAmerican classification of lymphoid neoplasms in 1994 and subsequently in the WHO classification [5].

E. Kehr $\cdot$ P. Stenzel $\cdot$ G. Xu $\cdot$ K. Kelemen $(\square)$

Department of Pathology, Oregon Health \& Science University,

L-471, 3181 Sam Jackson Park Road,

Portland, OR 97239-3098, USA

e-mail: Katikelemen99@yahoo.com
The classic initial clinical presentation of HSTL is hepatosplenomegaly, minimal or absent lymphadenopathy, and significant cytopenias. Thrombocytopenia is a constant feature and is frequently associated with anemia and/or leukopenia. A minor population of atypical lymphocytes can be identified in the peripheral blood of many patients; however, an overt leukemic presentation with leukocytosis due to large number of circulating $\mathrm{T}$ cell lymphoma is unusual at presentation. A number of sporadic reports of leukemic phase HSTL exist; however, the biological and clinical implications of leukemic phase have not been explored. We describe the case of a 65 -year-old man with leukemic phase of HSTL. The original diagnosis was established by flow cytometric analysis of the peripheral blood. Subsequent autopsy findings confirmed the diagnosis. We review 15 previously presented cases of leukemic phase HSTL and evaluate clinicopathologic features, response to therapy, and prognosis.

\section{Case presentation}

A 65-year-old man presented to a community hospital with 3 weeks history of daily fevers. His past medical history was noteworthy for a single vessel coronary artery bypass 14 years prior to admission; and his outpatient medications included rosuvastatin, metroprolol, lisinopril, and an aspirin. The patient had no history of autoimmune disease, immunosuppressive medication, or organ transplantation. On admission, physical examination showed splenomegaly and fever of $38.8^{\circ} \mathrm{C}$. Laboratory findings were remarkable for abnormal liver functions tests (AST of $351 \mathrm{U} / \mathrm{L}$; ALT of $121 \mathrm{U} / \mathrm{L}$; alkaline phosphatase of $350 \mathrm{U} / \mathrm{L}$; total bilirubin of $5.5 \mathrm{mg} / \mathrm{dL}$; and $\mathrm{LDH}$ of $4,329 \mathrm{U} / \mathrm{L}$ ) and pancytopenia (WBC, $2.6 \times 10^{9} / \mathrm{L}$; hematocrit, $26 \%$; platelets, $78 \times 10^{9} / \mathrm{L}$ ). 
Imaging revealed hepatosplenomegaly with no lymphadenopathy, and biopsies were performed of the liver and of the bone marrow. By report, the biopsies remained inconclusive as the liver biopsy showed zone 3 necrosis, and the bone marrow was interpreted as normal. An extensive infectious workup was negative.

He was transferred to our institution for further evaluation. In contrast to his prior pancytopenia, on the day of transfer, his CBC revealed a leukocytosis with a WBC of $18.6 \times 10^{9} / \mathrm{L}$ and with persistent anemia and thrombocyto- penia. His WBC differential showed $43 \%$ abnormal lymphocytes, $21 \%$ neutrophils, $14 \%$ bands, $21 \%$ small lymphocytes, and $1 \%$ monocytes.

Morphologic review of the peripheral blood smear (Fig. 1a, b) showed leukocytosis and a predominant population of large atypical lymphocytes with irregular nuclear contours, visible to prominent nucleoli, and variable amounts of basophilic cytoplasm with granules. In addition, the neutrophils showed toxic changes and a shift to immaturity with myelocytes and metamyelocytes. There
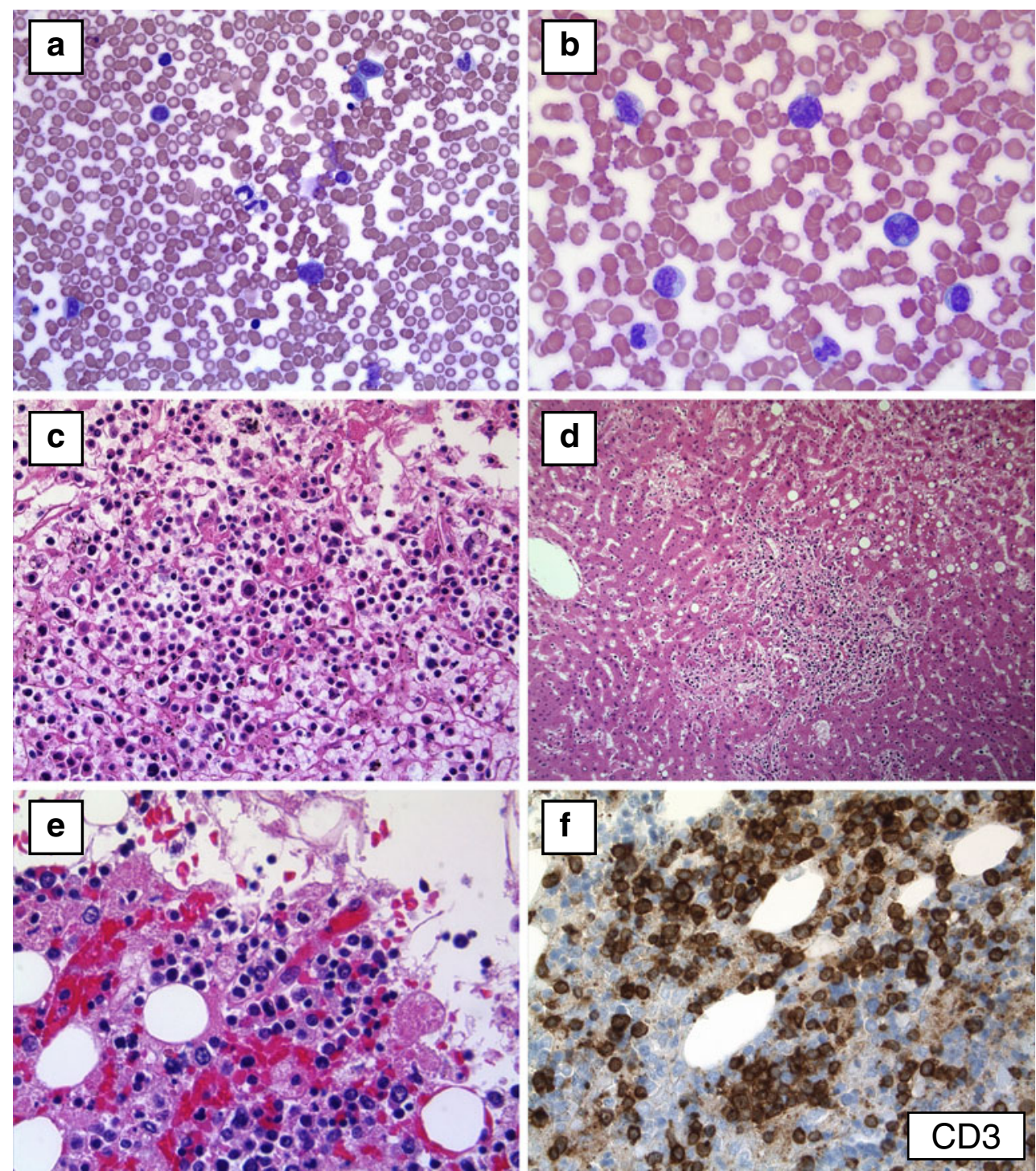

Fig. 1 a, b. Morphologic findings of the peripheral blood smear. There is a leukocytosis (WBC, $18.6 \times 10^{6} / \mathrm{L}$ ) with absolute lymphocytosis of $10.6 \times 10^{6} / \mathrm{L} .43 \%$ of the white blood cells are abnormal large lymphocytes showing variable amount of basophilic cytoplasm with cytoplasmic granules, irregular nuclei, and visible to prominent, often multiple nucleoli. The neutrophils show toxic changes and a shift to immaturity with myelocytes and metamyelocytes. In addition, there is a thrombocytopenia and a normochromic normocytic anemia with occasional nucleated red blood cells. c. Microscopic examination of the spleen, hematoxylin and eosin (H\&E). The red pulp sinusoids are

filled with a population of highly pleomorphic lymphocytes, characterized by large size, high nuclear/cytoplasmic ratios and hyperchromatic nuclei. d. H\&E-stained section of the liver shows focal macro-and microvesicular fatty change and infiltration of atypical lymphocytes in a primarily periportal pattern. Sinusoidal infiltrate is minimal. e, f H\&E-stained section of the bone marrow shows hypercellular marrow $(80 \%)$ with trilineage hematopoiesis and a sinusoidal and interstitial infiltrate of large, pleomorphic lymphocytes. Immunohistochemical staining with the T cell marker CD3 is positive and primarily highlights the sinusoidal content 
was a normochromic normocytic anemia, and occasional nucleated red blood cells were present. Platelets were also decreased.

Flow cytometric analysis of the peripheral blood was performed and identified an abnormal $\mathrm{T}$ cell population representing $44 \%$ of the white blood cells, remarkable for uniform positivity of the gamma-delta $\mathrm{T}$ cell receptor (TCR) and expression of CD2, CD3, CD56, and without expression of $\mathrm{CD} 5, \mathrm{CD} 4, \mathrm{CD} 8, \mathrm{CD} 7$, and the alpha-beta $\mathrm{T}$ cell receptor (flow cytometric findings are shown in Fig. 2.). Subsequently, PCR for the T cell receptor gamma region identified a clonal $\mathrm{T}$ cell population. Cytogenetic analysis of the peripheral blood by fluorescent in situ hybridization was negative for abnormalities of chromosomes 7 and 8 . The peripheral blood findings were interpreted as peripheral blood involvement by a $\mathrm{T}$ cell lymphoma/leukemia, most consistent with a gamma-delta hepatosplenic $\mathrm{T}$ cell lymphoma.

Over the subsequent $36 \mathrm{~h}$, the patient became progressively unstable with hypotension leading to acute renal failure. On hospital day 2, he developed chest pain accompanied by ST segment elevation on EKG, followed by cardiovascular arrest, and death. An autopsy was performed.

\section{Autopsy findings}

There was cardiomegaly (500 g), a single coronary bypass, severe occlusive atherosclerosis of the native left anterior
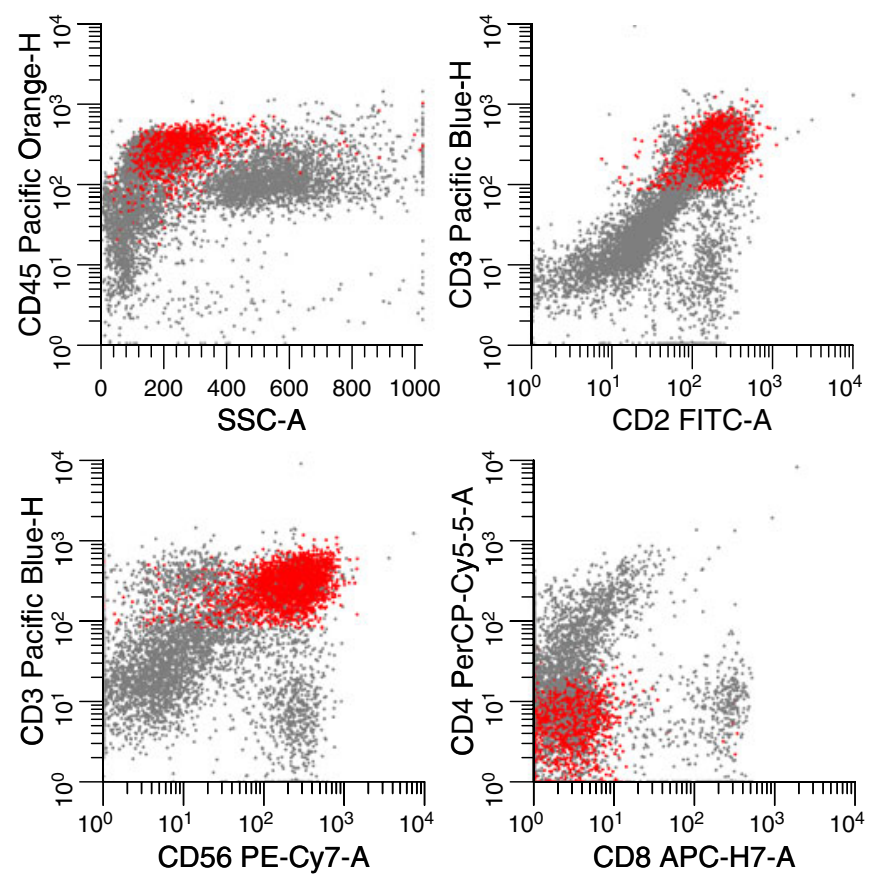

Fig. 2 Flow cytometric analysis of the peripheral blood identifies an immunophenotypically abnormal $\mathrm{T}$ cell population that comprises $43 \%$ of total leukocytes ( $76 \%$ of lymphocytes) and shows the descending artery, and a large scar in the left ventricle. No acute infarct was identified. Based on the history of chest pain associated with ST segment elevation and the evidence of chronic ischemic heart disease, the cause of death was assumed to be an acute myocardial infarct, too recent to produce histologic changes. The spleen weighed $980 \mathrm{~g}$ and showed a homogenously red cut surface. Histologic sections showed infiltration of the splenic red pulp by lymphocytes with abnormal pleomorphic morphology and primarily distributed within the sinusoids as shown in Fig. 1c. The lymphoid cells were similar cytologically to the lymphoma cells identified in the peripheral blood. The liver weighed 2,270 g with no localized masses. Microscopic analysis showed focal macrovesicular steatosis and periportal infiltration of abnormal medium to large pleomorphic lymphocytes (see Fig. 1d). The periportal lymphocytic infiltrate focally extended into adjacent sinusoids; however, the sinusoidal involvement was minimal. There was no lymphadenopathy identified in the autopsy. However, microscopic analysis of a randomly sampled $1 \mathrm{~cm}$ thoracic node showed partial sinusoidal involvement by the lymphoma. The bone marrow sections from rib and vertebrae showed a hypercellular bone marrow (cellularity estimated at $80 \%$ ) with trilineage hematopoiesis. Abnormal medium- to large-sized lymphocytes with irregular nuclei and a moderate cytoplasm were diffusely distributed throughout the marrow in a predominantly sinusoidal and interstitial pattern as shown in Fig. 1e. The pleomorphic lymphocytes filling the sinus-
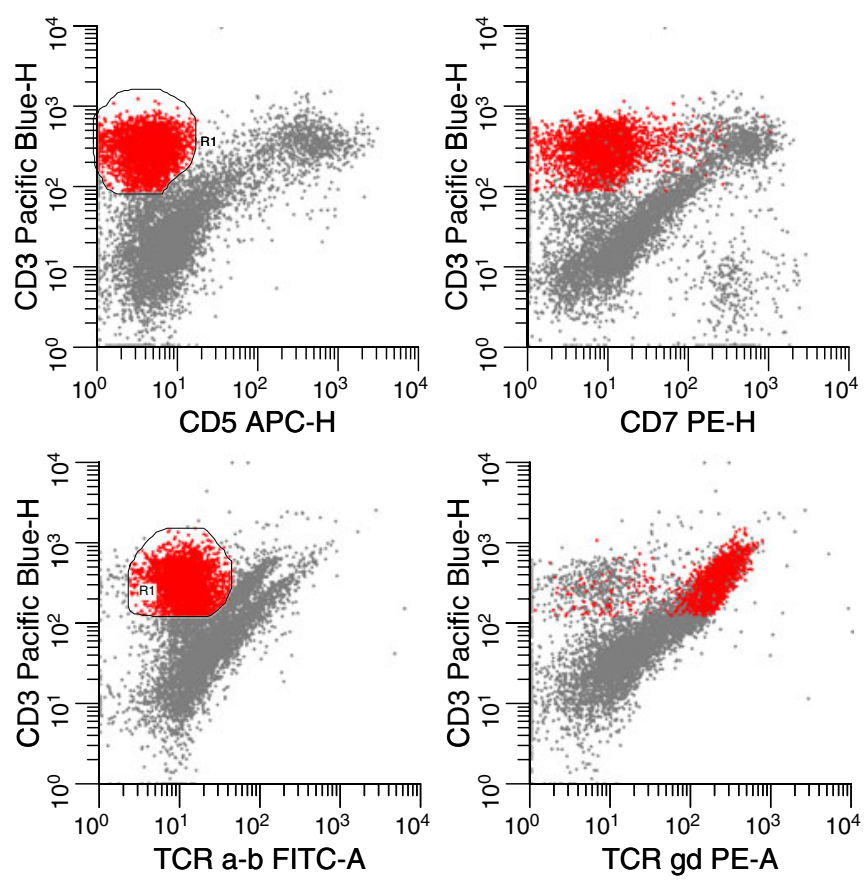

following immunophenotype: $\mathrm{CD} 2+, \mathrm{CD} 3+, \mathrm{CD} 45+, \mathrm{CD} 56+$, uniformly positive for gamma-delta $\mathrm{T}$ cell receptor and negative for $\mathrm{CD} 5$, $\mathrm{CD} 7, \mathrm{CD} 4, \mathrm{CD} 8$, and alpha beta $\mathrm{T}$ cell receptor 
oids are CD3 positive T cells (Fig. 1f). Histiocytes were not increased and hemophagocytosis was not identified. Gross examination of other major organs including brain, gastrointestinal system, kidneys, and testes was unremarkable. Microscopic examination showed presence of abnormal pleomorphic lymphocytes in the vascular spaces of all these organs; however, there was no evidence of tissue invasion or disruption of normal morphology seen in these organs. The prominence of the lymphoid cells in the vascular spaces most likely reflects the heavy involvement of the peripheral blood by lymphoma. Immunohistochemical staining was performed on sections from bone marrow, spleen, liver, and lymph node. The lymphocytic infiltrate in all these organs showed abnormal $\mathrm{T}$ cell immunophenotype expressing $\mathrm{CD} 2, \mathrm{CD} 3$, and $\mathrm{CD} 7$ and without expression of CD5, CD4, or CD8. The abnormal T cells were positive for expression of the cytotoxic ganuleassociated proteins granzyme B and TIA-1. EBER in situ hybridization for Epstein Barr virus (EBV) RNA was negative on the lymphoma cells. The immunophenotype reproduced the flow cytometric immunophenotypic findings observed in the peripheral blood with the exception that deletion of $\mathrm{CD} 7$ was not observed by immunohistochemistry.

\section{Discussion}

Hepatosplenic T cell lymphoma (HSTL) represents a diagnostic challenge, and the specific diagnosis is commonly delayed with a median of 60 days after presentation [2]. The classic combination of hepatosplenomegaly, fever, jaundice, and elevated liver enzymes often lead the diagnostic pursuit towards a viral infection, which is usually not confirmed [10]. Another common pitfall is misinterpretation of the isolated thrombocytopenia as idiopathic thrombocytopenic purpura $[1,3,7]$. The impact of the delay of the diagnosis on prognosis is not clear.

We report here the case of a 65-year-old Caucasian male, who initially presented with hepatosplenomegaly, no lymphadenopathy, elevated liver enzymes, fever, and pancytopenia. Despite a detailed workup, a specific diagnosis was not reached until 3 weeks after his original presentation, when he developed a leukocytosis and flow cytometric analysis identified an abnormal $\mathrm{T}$ cell population with an immunophenotype (CD2+, CD3+, CD45+, CD56+, negative for $\mathrm{CD} 5, \mathrm{CD} 7, \mathrm{CD} 4$, and $\mathrm{CD} 8$, uniform gammadelta TCR positive), highly suggestive of a gamma-delta HSTL. Within 2 days of the onset of leukocytosis, the patient died. The autopsy confirmed the diagnosis of in the spleen and in sections of the bone marrow in a primarily sinusoidal distribution. The liver was involved; however, the distribution of HSTL cells was somewhat unusual in that it was predominantly periportal, with minimal exten- sion into sinusoids. This pattern of liver infiltration, though rare, has been described in HSTL [17]. In situ hybridization was negative for EBV. The aberrant immunophenotype of the $\mathrm{T}$ cell lymphoma was reproduced by immunohistochemistry in sections of the bone marrow, spleen, and liver, and also demonstrated expression of two cytotoxic granule-associated proteins granzyme B and TIA-1. This pattern of cytotoxic granule-associated protein expression has been reported previously by several investigators in HSTL $[3,12,20]$.

The most remarkable feature of this case is the leukemic type peripheral blood involvement by HSTL and the impact of the peripheral blood involvement on the diagnostic workup. The biological and clinical implications of the leukemic phase of HSTL are not clear. In fact, exact criteria for a "leukemic phase" of HSTL do not exist. In our interpretation, a case of HSTL was considered leukemic if it is associated with absolute lymphocytosis of $4 \times 10^{6} / \mathrm{L}$ due to circulating $\mathrm{T}$ cell lymphoma cells. Using these criteria, we identified 15 previously published cases of leukemic HTCL in the literature. Demographic, clinicopathologic, and molecular features as well as clinical outcome of the 15 cases of HSTL with leukemic peripheral blood involvement are summarized in Table 1.

The age of patients with leukemic phase HSTL ranged form 8 to 65 years, (median of 39), with three patients in the pediatric age group $(8,9$, and 17$)$. The male to female ratio is $1: 1$. Just like HSTL in general, the majority of published cases were of gamma-delta TCR type (14/16) $[2,4,6,7,9,11-14,16-19]$; however, two cases of alphabeta TCR type with leukemic phase were also described in the literature [8]. Four of the 16 described cases occurred in transplant patients, including three kidney transplant and one bone marrow transplant patient [11, 14, 17, 19]. In this series, no cases of HSTL arising in the setting of immunosuppression, for reasons other than organ transplant, were identified. The onset of leukemic phase was variable in the course of the disease. In seven patients $(43 \%)$, the leukemic phase heralded a terminal event and occurred just prior to death, similar to our patient. In another $37 \%(6 / 16)$, the leukemic phase was already present at initial presentation and in two patients (12\%) developed in mid-phase, at 3 months, and 18 months after presentation, respectively.

From the morphologic point of view of the circulating $\mathrm{T}$ cell lymphoma cells, two different morphologic subtypes were described. In approximately two thirds of cases, the circulating $\mathrm{T}$ cell lymphoma cells were large, matureappearing, and pleomorphic, even bizarre, just like in our patient. In one third of cases, however, they appeared blastlike, with scant amount of cytoplasm, dispersed chromatin, and visible nucleoli. The two different morphologies might be important diagnostically, since they bring up different 


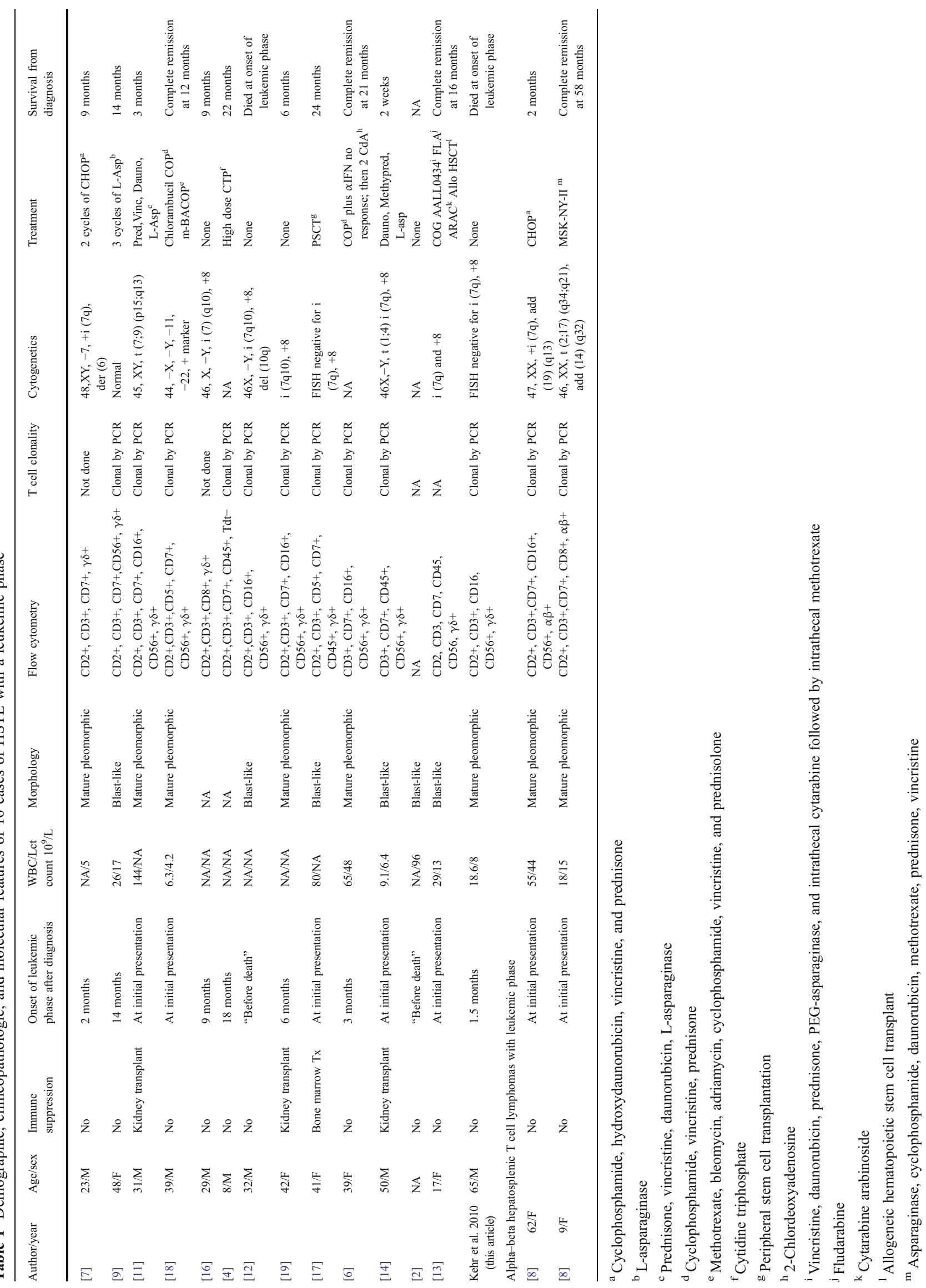


differential diagnostic entities, such as large granular lymphocyte leukemia, diffuse large B cell lymphoma in the mature pleomorphic type versus a $\mathrm{T}$ acute lymphoblastic leukemia or blastic NK cell leukemia in the blast-like type. Despite the two very different morphological forms, the flow cytometric immunophenotype appeared remarkably consistent and uniform: the circulating $\mathrm{T}$ cells are $\mathrm{CD} 2+, \mathrm{CD} 3+$, show downregulation of CD5 and/or CD7, commonly positive for NK cell markers CD16+ and CD56+, are bright CD45+, and most commonly double negative for CD4 and CD8. Rare cases, as expected, show CD8 positivity (1 of 13 gamma-delta TCR type and one of the two alpha-beta TCR-type HSTL were CD8 positive). The flow cytometric imunophenotype differentiates HSTL form other differential diagnostic entities, such as a large granular lymphocyte leukemia or blastic NK cell leukemia based on positivity for CD3 and negativity for EBV respectively; from $\mathrm{T}$ acute lymphoblastic leukemia based on lack of immature markers Tdt, CD10, and CD34, and the gamma-delta TCR differentiates it from other peripheral $\mathrm{T}$ cell lymphomas such as Sezary syndrome, $\mathrm{T}$ prolymphocytic leukemia, adult $\mathrm{T}$ cell leukemia/lymphoma, or peripheral $\mathrm{T}$ cell lymphoma not otherwise specified. Associated cytogenetic abnormalities of leukemic HSTL were in overlap with the cytogenetic abnormalities of HSTL without leukemic phase, isochromosome 7 with or without trisomy 8 being the most commonly detected abnormalities, and there was no evidence of clonal evolution or distinct chromosomal abnormality in the leukemic phase.

The prognosis of leukemic phase HSTL was poor but not significantly different from HSTL without leukemic involvement (range 0-58 months, median 9 months). Five cases with leukemic involvement achieved clinical remission greater than 12 months with treatment. This subset of patients was younger with a mean of 27 years of age. Interestingly, in all cases that were able to reach clinical remission, the leukemic peripheral blood involvement was present early in their disease (four of them were leukemic at initial presentation and one entered leukemic phase at 3 months after diagnosis). This suggests that the early peripheral blood involvement may have an impact on prognosis by providing an opportunity for quick noninvasive diagnosis by the means of peripheral blood flow cytometry.

Although leukemic peripheral blood involvement is rare in HSTL, a low level of circulating T cell lymphomas might be more common. Cooke et al. reviewed 19 cases of gamma-delta HTCL and found that 15 had some degree of peripheral blood involvement, though absolute lymphocytosis was rare [3]. Weidmann et al. desribe the presence of lymphoma cells in peripheral blood in 16 of 32 patients $(50 \%)$ [17]. Vega et al. reported eight patients with HSTL, and although none of them had absolute lymphocytosis, five of them had evidence of low-level peripheral blood involvement [15]. Similarly, in a series of 21 patients with HSTL, Belhadj et al. observed ten cases with peripheral blood involvement, including one patient with leukemic phase, and seven additional subjects with low level of circulating $\mathrm{T}$ cell lymphoma [2]. Considering the remarkable stability of flow cytometric immunophenotype, even low-level circulating lymphoma cells may provide an opportunity for a fast noninvasive and very specific diagnosis, if peripheral blood flow cytometry is attempted.

In summary, leukemic phase of HSTL is rare; however, low level of circulating $\mathrm{T}$ cell lymphoma are commonly present in the peripheral blood, with a reported frequency between $40-80 \%$ of cases. The highly characteristic immunophenotype enables a quick, noninvasive diagnosis of HSTL; yet, flow cytometry of peripheral blood has not been acknowledged as a primary tool in the diagnostic algorithm of HSTL, especially in cases where leukocytosis is not present. We conclude that low-level peripheral blood involvement is an important feature of HSTL, despite the usual presentation with pancytopenia. Morphologic review of the peripheral blood smear and flow cytometric analysis of the peripheral blood should be incorporated into the early diagnostic workup and might facilitate an earlier diagnosis for patients whose differential diagnosis includes HSTL.

Conflict of interest The authors declare that they have no conflict of interest.

\section{References}

1. Alonsozana EL, Stamberg J, Kumar D, Jaffe ES, Medeiros LJ, Frantz C, Schiffer CA, O'Connell BA, Kerman S, Stass SA, Abruzzo LV (1997) Isochromosome 7q: the primary cytogenetic abnormality in hepatosplenic gamma delta $\mathrm{T}$ cell lymphoma. Leukemia 11:1367-1372

2. Belhadj K, Reyes F, Farcet JP, Tilly H, Bastard C, Angonin R, Deconinck E, Charlotte F, Leblond V, Labouyrie E, Lederlin P, Emile JF, Delmas-Marsalet B, Arnulf B, Zafrani ES, Gaulard P (2003) Hepatosplenic gamma delta T-cell lymphoma is a rare clinicopathologic entity with poor outcome: report on a series of 21 patients. Blood 102:4261-4269

3. Cooke CB, Krenacs L, Stetler-Stevenson M, Greiner TC, Raffeld M, Kingma DW, Abruzzo L, Frantz C, Kaviani M, Jaffe ES (1996) Hepatosplenic T-cell lymphoma: a distinct clinicopathologic entity of cytotoxic gamma delta T-cell origin. Blood 88:4265-4274

4. Garcia-Sanchez F, Menarguez J, Cristobal E, Cantalejo A, Gil J, Algara P, Vicario JL (1995) Hepatosplenic gamma-delta T-cell malignant lymphoma: report of the first case in childhood, including molecular minimal residual disease follow-up. $\mathrm{Br} \mathrm{J}$ Haematol 90:943-946

5. Gaulard P, Jaffe ES, Krenacs L, Macon WR (2008) Hepatosplenic T-cell lymphoma. In: Swerdlow SH, Campo E, Harris NL, Jaffe ES, Pileri SA, Stein H, Thiele J, Vardiman JW (eds) World Health Organization classification of tumors of haematopoietic and lymphoid tissues. International Agency for Research on Cancer, Lyon, pp 292-293 
6. Gopcsa L, Banyai A, Tamaska J, Karadi A, Matolcsy A, Paloczi K (2002) Hepatosplenic gamma delta T-cell lymphoma with leukemic phase successfully treated with 2-chlorodeoxyadenosine. Haematologia (Budap) 32:519-527

7. Lin MT, Shen MC, Su IJ, Tien HF, Chen YC, Hsu PN, Chang CS, Wang CH (1991) Peripheral T gamma/delta lymphoma presenting with idiopathic thrombocytopenic purpura-like picture. $\mathrm{Br} \mathrm{J}$ Haematol 78:280-282

8. Macon WR, Levy NB, Kurtin PJ, Salhany KE, Elkhalifa MY, Casey TT, Craig FE, Vnencak-Jones CL, Gulley ML, Park JP, Cousar JB (2001) Hepatosplenic alphabeta T-cell lymphomas: a report of 14 cases and comparison with hepatosplenic gammadelta T-cell lymphomas. Am J Surg Pathol 25:285-296

9. Mastovich S, Ratech H, Ware RE, Moore JO, Borowitz MJ (1994) Hepatosplenic T-cell lymphoma: an unusual case of a gamma delta T-cell lymphoma with a blast-like terminal transformation. Hum Pathol 25:102-108

10. Ohno T, Komada F, Yamaguchi M, Oka K, Nishii K, Tsuda M, Katsuta K, Yamaguchi T, Kita K, Shirakawa S (1993) Gamma/ delta T-cell lymphoma with hepatosplenomegaly: report of a case. Int J Hematol 57:269-276

11. Ross CW, Schnitzer B, Sheldon S, Braun DK, Hanson CA (1994) Gamma/delta T-cell posttransplantation lymphoproliferative disorder primarily in the spleen. Am J Clin Pathol 102:310-315

12. Salhany KE, Feldman M, Kahn MJ, Peritt D, Schretzenmair RD, Wilson DM, DiPaola RS, Glick AD, Kant JA, Nowell PC, Kamoun M (1997) Hepatosplenic gammadelta T-cell lymphoma: ultrastructural, immunophenotypic, and functional evidence for cytotoxic T lymphocyte differentiation. Hum Pathol 28:674-685

13. Shafer E, Chen A, Arceci RJ (2009) Sustained first remission in an adolescent with hepatosplenic T-cell lymphoma treated with Tcell leukemia induction, nucleoside analog-based consolidation, and early hematopoietic stem cell transplant. Pediatr Blood Cancer 53:1127-1129

14. Steurer M, Stauder R, Grunewald K, Gunsilius E, Duba HC, Gastl G, Dirnhofer S (2002) Hepatosplenic gammadelta-T-cell lymphoma with leukemic course after renal transplantation. Hum Pathol 33:253-258

15. Vega F, Medeiros LJ, Bueso-Ramos C, Jones D, Lai R, Luthra R, Abruzzo LV (2001) Hepatosplenic gamma/delta T-cell lymphoma in bone marrow. A sinusoidal neoplasm with blastic cytologic features. Am J Clin Pathol 116:410-419

16. Wang CC, Tien HF, Lin MT, Su IJ, Wang CH, Chuang SM, Shen MC, Liu CH (1995) Consistent presence of isochromosome $7 \mathrm{q}$ in hepatosplenic $\mathrm{T}$ gamma/delta lymphoma: a new cytogenetic-clinicopathologic entity. Genes Chromosom Cancer 12:161-164

17. Weidmann E (2000) Hepatosplenic T cell lymphoma. A review on 45 cases since the first report describing the disease as a distinct lymphoma entity in 1990. Leukemia 14:991-997

18. Wong KF, Chan JK, Matutes E, McCarthy K, Ng CS, Chan $\mathrm{CH}$, Ma SK (1995) Hepatosplenic gamma delta T-cell lymphoma. A distinctive aggressive lymphoma type. Am J Surg Pathol 19:718726

19. Wu H, Wasik MA, Przybylski G, Finan J, Haynes B, Moore H, Leonard DG, Montone KT, Naji A, Nowell PC, Kamoun M, Tomaszewski JE, Salhany KE (2000) Hepatosplenic gamma-delta T-cell lymphoma as a late-onset posttransplant lymphoproliferative disorder in renal transplant recipients. Am J Clin Pathol 113:487-496

20. Yamaguchi M, Ohno T, Nakamine H, Oka K, Matsuzuka F, Miwa H, Shiku H, Kimura N, Nanba K, Kita K (1999) Gamma delta T-cell lymphoma: a clinicopathologic study of 6 cases including extrahepatosplenic type. Int J Hematol 69:186-195 